\title{
Structural and Optical Properties of Amorphous and Crystalline GeSn Layers on Si
}

\author{
Ruben R. Lieten, ${ }^{\mathrm{a}, \mathrm{b}, \mathrm{c}, *, \mathrm{z}}$ Claudia Fleischmann, ${ }^{\mathrm{b}, \mathrm{d}}$ Sven Peters, ${ }^{\mathrm{e}}$ Nuno M. Santos, ${ }^{\mathrm{d}}$ \\ Ligia Marina Amorim, ${ }^{\mathrm{d}}$ Yosuke Shimura ${ }^{\mathrm{b}, *}$ Noriyuki Uchida, ${ }^{\mathrm{f}}$ Tatsuro Maeda, ${ }^{\mathrm{e}}$ S. Nikitenko, ${ }^{\mathrm{g}}$ \\ Thierry Conard, ${ }^{\mathrm{b}}$ Jean-Pierre Locquet, ${ }^{\mathrm{a}}$ Kristiaan Temst, ${ }^{\mathrm{d}}$ and André Vantomme ${ }^{\mathrm{d}}$ \\ ${ }^{a}$ Solid State Physics and Magnetism, Department of Physics and Astronomy, KU Leuven, 3001 Leuven, Belgium \\ ${ }^{b}$ IMEC, 3001 Leuven, Belgium \\ ${ }^{c}$ Entegris, 3001 Leuven, Belgium \\ ${ }^{d}$ Instituut voor Kern-en Stralingsfysica, KU Leuven, 3001 Leuven, Belgium \\ e SENTECH Instruments GmbH, D-12489 Berlin, Germany

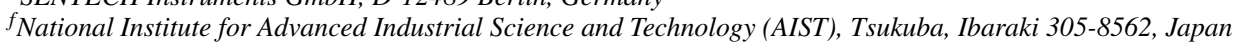 \\ ${ }^{g}$ Netherlands Organization for Scientific Research (NWO), DUBBLE@ESRF, 38043 Grenoble, France
}

\begin{abstract}
We have investigated the structural and optical properties of metastable amorphous and crystalline GeSn layers on Si substrates The as-deposited amorphous layers crystallize during annealing at $500^{\circ} \mathrm{C}$. This transition leads to a significant change in the local environment of the $\mathrm{Sn}$ atoms and in the optical properties. The Ge-Sn bond length is decreased after crystallization. The as-deposited GeSn layers, with nominal $4.5 \%$ and $11.3 \%$ Sn content, do not show Sn segregation. For the crystallized GeSn with nominal $4.5 \%$, the $\mathrm{Sn}$ appears to be substitutional, as no $\mathrm{Sn}$ clustering was observed. However, for the crystallized GeSn with nominal $11.3 \% \mathrm{Sn}$, Sn segration and the presence of $\beta-\mathrm{Sn}$ is observed by EXAFS. A method to suppress Sn segregation and increase the substitutional Sn concentration is discussed. Furthermore, we determined the optical properties of amorphous and crystalline GeSn with nominal $4.5 \% \mathrm{Sn}$. The bandgap energy decreases significantly from $0.89 \mathrm{eV}$ (1392 nm) $\pm 0.05 \mathrm{eV}$ for the amorphous layer to $0.52 \mathrm{eV}(2383 \mathrm{~nm}) \pm 0.05 \mathrm{eV}$ for crystalline $\mathrm{GeSn}$, leading to significant reduction in penetration depth.

(C) The Author(s) 2014. Published by ECS. This is an open access article distributed under the terms of the Creative Commons Attribution Non-Commercial No Derivatives 4.0 License (CC BY-NC-ND, http://creativecommons.org/licenses/by-nc-nd/4.0/), which permits non-commercial reuse, distribution, and reproduction in any medium, provided the original work is not changed in any way and is properly cited. For permission for commercial reuse, please email: oa@electrochem.org. [DOI: 10.1149/2.0091412jss] All rights reserved.
\end{abstract}

Manuscript submitted August 22, 2014; revised manuscript received October 2, 2014. Published October $11,2014$.

$\mathrm{Ge}_{1-\mathrm{x}} \mathrm{Sn}_{\mathrm{x}}(\mathrm{GeSn})$ has been predicted to exhibit carrier mobilities exceeding those of both $\mathrm{Ge}$ and $\mathrm{Si}^{1}{ }^{1}$ making it interesting as an alternative channel material in high-speed Si complementary metal-oxide semiconductor (CMOS) technology. An increase in carrier mobility for $\mathrm{GeSn}$ with respect to Ge has recently been demonstrated in a MOSFET device by Gupta et al. ${ }^{2}$ In addition, GeSn exhibits a direct bandgap for a Sn concentration of $\pm 6.5 \%$, and is therefore promising for optical applications. ${ }^{3-6}$ While previous GeSn channel transistors were predominantly fabricated on Ge substrates, ${ }^{2,7}$ integration into $\mathrm{Si}$ is preferred for CMOS compatibility. The epitaxial growth of GeSn on Si substrates poses several challenges however. The limited solubility of $\mathrm{Sn}$ in $\mathrm{Ge}(0.5 \%)$ and the large lattice mismatch $(>4 \%)$ between $\mathrm{GeSn}$ and Si lead to compositional fluctuations, Sn segregation, and significant roughening. ${ }^{8-14}$

Recently, we have demonstrated the fabrication of singlecrystalline GeSn layers on $\mathrm{Si}(111)$ substrates with excellent structural quality by solid phase epitaxy (SPE) of amorphous GeSn layers. ${ }^{15}$ The key breakthrough is the limitation of the adatom surface mobility during deposition by introducing inert gas species, ${ }^{16}$ leading to a homogeneous and amorphous GeSn layer with a high Sn content on $\mathrm{Si}^{15}$ Subsequent thermal annealing induces SPE and leads to singlecrystalline GeSn. The excellent physical properties of these layers have been demonstrated in biaxially tensile strained depletion-mode $\mathrm{GeSn}(111)$ pMOSFETs with a TaN/ $\mathrm{Al}_{2} \mathrm{O}_{3}$ gate stack and NiGeSn metal source/drain (S/D) ${ }^{17}$ which show well-behaved junctionless GeSn pMOSFET operation. Despite this technological adavancement, little is known about the mechanisms that govern GeSn SPE, and so far little account has been taken for their physicochemical properties such as atomic structure, atom spacing, composition, etc. The aim of this study is to assess the fundamental aspects of SPE by measuring the structural properties of the as deposited amorphous GeSn film as well as the annealed film after SPE (crystallization). In particular, we investigate the local environment around the $\mathrm{Sn}$ atoms in the $\mathrm{GeSn}$ layers using Sn K-edge (29200 eV) X-ray absorption spectroscopy (XAS). This

\footnotetext{
*Electrochemical Society Active Member.
}

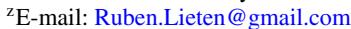

is complemented by Rutherford backscattering spectrometry (RBS), $\mathrm{X}$-ray diffraction (XRD), and Raman spectroscopy measurements to assess the crystalline quality, Sn content, layer thickness, composition fluctuations, and strain distribution. In view of application, we finally performed optical measurements on the amorphous and crystallized GeSn layers to extract the bandgap, which can be directly linked with the structural properties in strained GeSn films.

\section{Experimental}

Si substrates with (111) orientation and resistivity of 2000-4000 Ohm.cm were chemically cleaned to remove surface contamination and loaded into an ultrahigh vacuum (UHV) chamber. Amorphous GeSn (a-GeSn) layers, with thicknesses between $37 \mathrm{~nm}$ and $65 \mathrm{~nm}$, were deposited onto the $\mathrm{Si}(111)$ substrates. Ultra-pure $\mathrm{Ge}$ and $\mathrm{Sn}$ were evaporated from separate effusion cells in the UHV chamber (base pressure of $2 \times 10^{-10}$ Torr). The adatom surface mobility was reduced by exposing the substrate to a $\mathrm{N}_{2}$ flux with a beam equivalent pressure (BEP) of $\sim 2 \times 10^{-5}$ Torr and using a deposition temperature of around $10^{\circ} \mathrm{C}^{15,16}$ For a nominal $\mathrm{Sn}$ content of $4.5 \%(11.3 \%)$ we used BEPs of $9.0 \times 10^{-9}\left(2.25 \times 10^{-8}\right)$ Torr and $1.33 \times 10^{-7}(1.24$ $\times 10^{-7}$ ) Torr for $\mathrm{Sn}$ and $\mathrm{Ge}$, respectively. Subsequent annealing at $500^{\circ} \mathrm{C}$ for $1 \mathrm{~min}$ in $\mathrm{N}_{2}$ ambient transforms the amorphous GeSn into a single-crystalline layer by SPE. ${ }^{15}$ A Ga concentration of around 2.6 $\times 10^{18} \mathrm{~cm}^{-3}$ is present in the layer because the particular Ge source material used in these experiments contains a small concentration of Ga atoms, as observed by secondary ion mass spectrometry (SIMS). Sample information is summarized in Table I. Amorphous GeSn layers with nominal $4.5 \%$ and $11.3 \% \mathrm{Sn}$ are indicated as a-GeSn(4.5\%) and a-GeSn(11.3\%), respectively, whereas crystalline GeSn layers with nominally $4.5 \%$ and $11.3 \% \mathrm{Sn}$ are indicated as c-GeSn(4.5\%) and c-GeSn(11.3\%), respectively.

\section{Results}

In the following sections, we investigate the structural properties of the amorphous and crystallized GeSn layers. First, the Sn content, composition profile and thickness have been investigated by RBS 
Table I. Sample information of amorphous and crystallized GeSn layers on Si(111), and summary of Raman, XRD, RBS and EXAFS results.

\begin{tabular}{|c|c|c|c|c|}
\hline & $\mathrm{a}-\mathrm{GeSn}(4.5 \%)$ & $\mathrm{c}-\mathrm{GeSn}(4.5 \%)$ & $\mathrm{a}-\mathrm{GeSn}(11.3 \%)$ & $\mathrm{c}-\mathrm{GeSn}(11.3 \%)$ \\
\hline $\mathrm{Sn} /(\mathrm{Ge}+\mathrm{Sn})$ flux ratio $(\%)$ & 4.5 & 4.5 & 11.3 & 11.3 \\
\hline Structure & amorphous & crystalline & amorphous & crystalline \\
\hline Thickness (nm) & 63 & 63 & 64 & 60 \\
\hline Sn from RBS $(\%)$ & $5.0 \pm 0.5$ & $4.9 \pm 0.5$ & $12.9 \pm 0.5$ & $13.7 \pm 0.5$ \\
\hline Sn from RSM $(\%)$ & 1 & $4.1 \pm 0.7$ & 1 & $3.9 \pm 0.7$ \\
\hline In-plane spacing from XRD $(\AA)$ & I & $5.709 \pm 0.001$ & I & $5.695 \pm 0.001$ \\
\hline Average Ge-Ge and Ge-Sn bond length from XRD $(\AA)$ & I & $2.472 \pm 0.001$ & l & $2.466 \pm 0.001$ \\
\hline In-plane strain $(\%)$ & l & $+0.29 \pm 0.02$ & l & $+0.07 \pm 0.02$ \\
\hline Raman shift $\left(\mathrm{cm}^{-1}\right)$ & $-28.0 \pm 0.1$ & $-5.1 \pm 0.1$ & $-31.3 \pm 0.1$ & $-5.5 \pm 0.1$ \\
\hline Sn-Ge distance $(\AA)$ & $2.65 \pm 0.05$ & $2.60 \pm 0.05$ & $2.64 \pm 0.05$ & $2.59 \pm 0.05$ \\
\hline$\beta$-Sn-Sn observed by EXAFS & 1 & 1 & 1 & yes \\
\hline
\end{tabular}

measurements using a $1.57 \mathrm{MeV} 4 \mathrm{He}^{+}$beam and two solid state detectors positioned at a scattering angle of $168^{\circ}$ and $105^{\circ}$, respectively see Table I. Analysis of the measured RBS spectrum shows a Sn concentration of $5.0 \%, 4.9 \%, 12.9 \%$ and $13.7 \%$ for the a-GeSn $(4.5 \%)$, $\mathrm{c}-\mathrm{GeSn}(4.5 \%)$, a-GeSn(11.3\%) and c-GeSn(11.3\%) sample, respectively, which is close to the nominal value. The thickness of all samples is comparable: $66 \mathrm{~nm}, 63 \mathrm{~nm}, 64 \mathrm{~nm}, 60 \mathrm{~nm}$ for a-GeSn(4.5\%), c$\operatorname{GeSn}(4.5 \%)$, a-GeSn(11.3\%) and c-GeSn(11.3\%), respectively. For the layers with nominal $4.5 \% \mathrm{Sn}$, both the as-deposited amorphous GeSn layer and the annealed crystalline sample, show a uniform Sn profile, see Figure 1. The samples with nominal $11.3 \% \mathrm{Sn}$, both the as-deposited and the annealed sample, show a lower Sn composition at the surface. The annealed c-GeSn(11.3\%) sample shows higher $\mathrm{Sn}$ content at the interface with the Si substrate, indicating segregation of Sn during the annealing step.

The lattice spacings are accurately measured by high-resolution reciprocal space map (RSM) XRD measurements, around the (331) reflection. From these measurements the elastic strain and composition of the crystalline GeSn layers with nominal $4.5 \%$ and $11.3 \% \mathrm{Sn}$ have been determined, taking into account the elastic moduli of $\mathrm{Ge}$ and $\mathrm{Sn},{ }^{15}$ as listed in Table I. The corresponding Sn content, in-plane lattice spacing and in-plane strain of sample c-GeSn $(4.5 \%)$ and sample c-GeSn $(11.3 \%)$ amount to $4.1 \%, 5.709 \AA$ and $+0.29 \%$, as well as $3.9 \%, 5.695 \AA$ and $+0.07 \%$, respectively. The measurement error on the composition, lattice spacing and in-plane strain are $\pm 0.1 \%$, $\pm 0.001 \AA$ and $\pm 0.02 \%$, respectively. Additionally, there is a parameter error ((the real elastic constants are different than assumed) and model error (Vegard's law is not completely valid), which underestimates the Sn composition with $0.7 \%$ for crystalline $\mathrm{GeSn}$ with $4.5 \%$ Sn content as measured by RBS. ${ }^{15}$

As the Sn content and the lattice spacing are comparable for both samples, we infer that in sample c-GeSn(11.3\%) a large fraction of

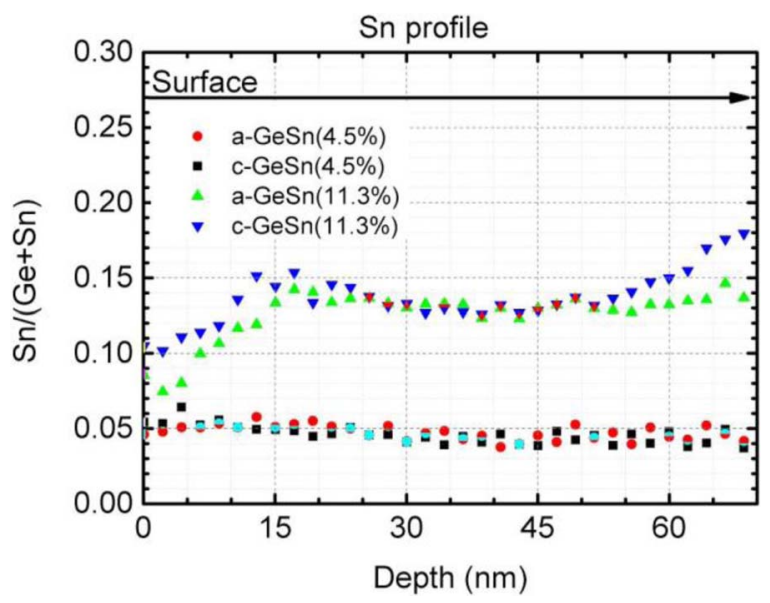

Figure 1. Sn composition profile as function of depth from the sample surface, calculated from RBS measurements. the $\mathrm{Sn}$ atoms is not incorporated substitutionally in the Ge diamond crystal structure. This is in agreement with the conclusions drawn from the Raman measurements in the following section.

In order to assess the structural quality, Sn content, and strain distribution in the GeSn samples, we have performed Raman measurements using a $488 \mathrm{~nm}$ excitation source, see Figure $2 \mathrm{a}$ and $2 \mathrm{~b}$ and Table I. A bulk Ge wafer was measured as a reference (green solid lines), showing an intense and sharp Ge-Ge phonon scattering peak at $301 \mathrm{~cm}^{-1}$ with full width at half maximum (FWHM) of $5.7 \mathrm{~cm}^{-1}$. For the amorphous a-GeSn samples with $(4.5 \%)$ and a-GeSn(11.3\%) samples (red solid lines) a weak and broad feature is visible, which is shifted to lower wavenumbers with respect to the bulk Ge-Ge phonon peak by $-28.0 \mathrm{~cm}^{-1}$ and by $-31.3 \mathrm{~cm}^{-1}$, respectively. After annealing (blue solid lines), the structural order increases significantly in both samples, as follows from the increase in intensity and the decrease in peak width of the phonon scattering peaks. When compared to the amorphous samples, the peak shift of the Ge-Ge phonon peak (with respect to bulk $\mathrm{Ge}$ ) is smaller after crystallization. In general, the peak shift originates from the incorporation of $\mathrm{Sn}$ into the Ge lattice as well as from the tensile strain caused by the thermal mismatch between $\mathrm{GeSn}$ and Si. This thermal strain is introduced in the crystallized samples during cooling from the crystallization temperature $\left(500^{\circ} \mathrm{C}\right)$ to room temperature. For the as-deposited samples, no thermal strain is introduced as the deposition temperature $\left(10^{\circ} \mathrm{C}\right)$ is close to RT. Lin et al. have shown that the peak frequency shift $\Delta \omega$ increases linearly with the Sn fraction $x$ and the strain $\varepsilon_{\mathrm{a}}: \Delta \omega=\omega_{G e S n}-\omega_{G e}=\alpha \cdot x+\beta \cdot \varepsilon_{a}$ with $\alpha=-82 \mathrm{~cm}$ and $\beta=-563 \mathrm{~cm}^{-1} \cdot{ }^{18}$ Applying this equation to our system with a Sn content of $4.5 \%$ and a tensile strain of $+0.34 \%$, as measured by RBS/XRD and reported in reference 15 , respectively, we obtain a shift of $-5.6 \mathrm{~cm}^{-1}$, close to our experimental value of $-5.1 \mathrm{~cm}^{-1}$ for the c-GeSn(4.5\%) sample. On the other hand, a Sn concentration of $11.3 \%$ should yield a peak shift of $-10.1 \mathrm{~cm}^{-1}$, based on the above equation and assuming a strain of $+0.15 \% .{ }^{15}$ This calculated peak shift is much higher than the measured shift of $-5.5 \mathrm{~cm}^{-1}$ for the c-GeSn $(11.3 \%)$ sample. Also, the measured shift is comparable for both crystalline samples, c-GeSn(4.5\%) and c-GeSn(11.3\%), which suggests a similar Ge-Ge bond length in both samples despite the difference in Sn concentration as measured with RBS. Hence, only a fraction of the supplied $\mathrm{Sn}$ is incorporated substitutionally in the c-GeSn(11.3\%) sample. Because of the limited sensitivity of Raman spectroscopy and the low Sn concentration in our samples, we do not observe Sn-Sn phonon scattering, which is predicted to occur around $160 \mathrm{~cm}^{-1} .{ }^{19}$ The presence of Sn clusters will however be confirmed by XAS measurements in the following section.

Both crystallized samples exhibit in-plane biaxial tensile strain, which follows from the thermal mismatch between GeSn and Si. For sample c-GeSn(4.5\%), the measured tensile strain of $+0.29 \%$ is larger than the theoretical thermal strain of $+0.16 \%$ for cooling from $500^{\circ} \mathrm{C}$ to RT, calculated using the thermal expansion coefficients of Si (2.6 $\left.\times 10^{-6 \circ} \mathrm{C}^{-1}\right)$ and $\mathrm{Ge}_{0.955} \mathrm{Sn}_{0.045}\left(5.8 \times 10^{-6 \circ} \mathrm{C}^{-1}\right) .{ }^{15} \mathrm{As} \mathrm{HR}-\mathrm{XRD}$ is only suited to determine the lattice spacing, hence the bond length information in crystalline layers, we have performed additional XAS measurements to assess the structural properties of the amorphous 

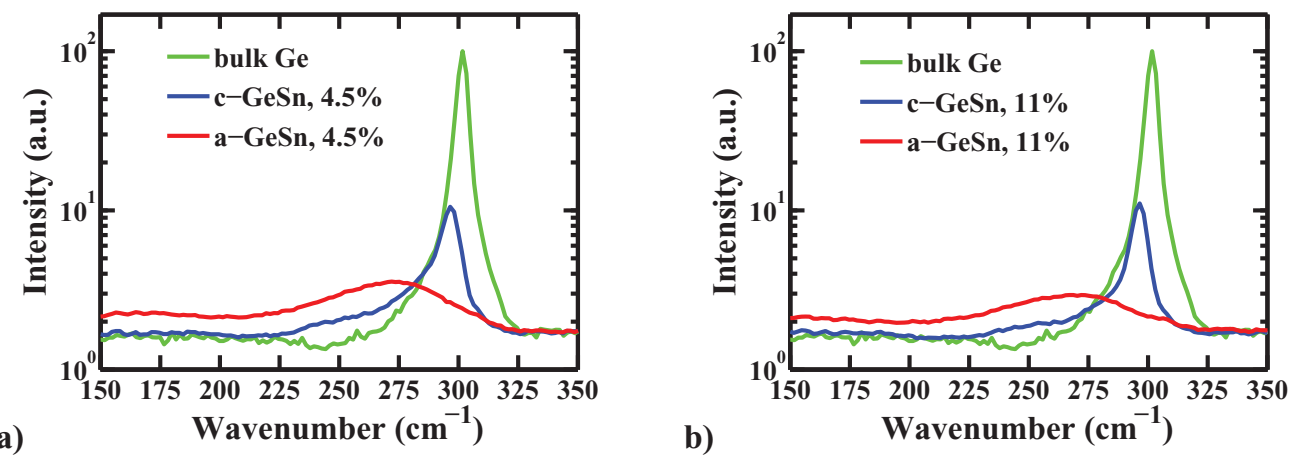

Figure 2. Raman measurements of amorphous and crystallized GeSn layers for nominal a) $4.5 \%$ and b) $11.3 \%$ Sn content. A reference measurement of bulk Ge is also included.

layers before SPE. This method also complements the Raman and HR-XRD measurements on the crystalline samples, due to its high sensitivity and element-specificity.

The near-edge X-ray absorption fine structure (XANES) and the extended X-ray absorption fine structure (EXAFS) measurements have been performed around the Sn K-edge $(29.2 \mathrm{keV})$ at the DutchBelgian CRG (DUBBLE, BM26A) beamline using bending magnet radiation from the European Synchrotron Radiation Facility (ESRF). EXAFS is used to determine the distances, coordination number and neighboring species of the absorbing atom, whereas XANES is strongly sensitive to the oxidation state and coordination chemistry of the absorbing atom.

All spectra presented in this work were recorded in fluorescence mode using a 9-element monolithic Ge detector positioned at $90^{\circ}$ with respect to the incoming X-ray beam. XAS measurements were performed at grazing incidence angles $\left(\leq 1^{\circ}\right)$ to increase the illuminated surface area of the GeSn thin layer and to reduce the background signal arising from X-ray scattering and diffraction from the crystalline substrate. A metallic Sn foil was used as a reference. Data processing and analysis was done with the ATHENA and ARTEMIS packages, ${ }^{20}$ the graphical interfaces to IFEFFIT 1.2.11c. ${ }^{21}$ Theoretical fitting standards were computed by FEFF8. ${ }^{22,23}$ Data were recorded in k-range up to $12 \AA^{-1}$ and data fitting was performed in R-space between 1.8 and $3.0(3.5) \AA$.

The magnitude of the Fourier transform (FT) of the k-weighted EXAFS oscillation $\chi(\mathrm{k})$ is shown in Figure $3 \mathrm{a}$ for the $65 \mathrm{~nm}$ amorphous a-GeSn $(4.5 \%)$ and the $63 \mathrm{~nm}$ crystalline c-GeSn(4.5\%) samples. Both spectra exhibit one distinct peak originating from scattering at the first
Sn coordination shell. The fact that the peak intensity is higher for the annealed than for the as-grown sample indicates an enhanced ordering in the annealed, crystalline sample. A closer look at this spectrum reveals a second, small peak at a distance of about $3.5 \AA$ (not corrected for the scattering phase shift $\Delta$ ) from the Sn absorber atom. Although this feature is only slightly higher than the background signal it may be an additional indication for an increased local order in the annealed c-GeSn(4.5\%) film compared to the as-grown structure. In agreement with this, the XRD and Raman measurements show a significant increase in long-range ordering compared to the amorphous samples. The fact that the EXAFS data of the annealed c-GeSn(4.5\%) sample do not exhibit pronounced scattering peaks from the second (or higher) coordination shell may be an indication for minor disruption in the local order around the $\mathrm{Sn}$ atoms at medium distance (up to $5 \AA$ away from the $\mathrm{Sn}$ atoms).

From a comparison of the EXAFS data obtained on the $4.5 \%$ $\mathrm{GeSn}$ samples to a metallic $\mathrm{Sn}$ reference sample, both indicated in Figure 3, we infer that the first peak in the FT, around $2.4 \AA$ (not corrected for the scattering phase shift delta) is not linked to $\mathrm{Sn}-\mathrm{Sn}$ scattering and thus arises from Sn-Ge scattering. In other words, the EXAFS measurements give no indication for Sn agglomeration in the as-deposited, amorphous sample (sample a-GeSn(4.5\%)) nor in the annealed, crystalline sample (sample c-GeSn(4.5\%)). The EXAFS data of the crystalline sample were fitted (solid line, Figure 3) with a Sn-Ge single scattering path (degeneracy $\mathrm{N}<4$ ) with the absorber atom ( $\mathrm{Sn}$ ) on a substitutional lattice site in a Ge diamond crystal structure. The coordination number is slightly reduced compared to the ideal bulk structure, from which we infer a minor local disorder
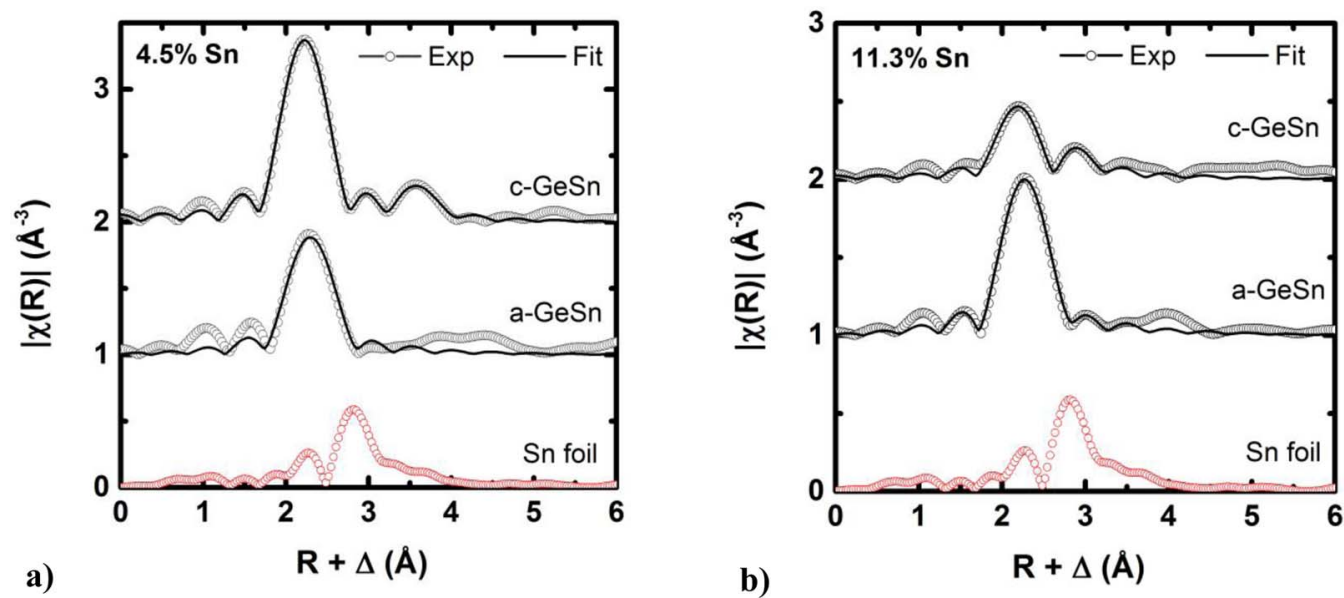

Figure 3. EXAFS data (not corrected for scattering phase shift $\Delta$ ) for crystalline GeSn and amorphous GeSn with nominally a) $4.5 \%$ and b) $11.3 \%$ Sn concentration. EXAFS data for a metallic Sn reference foil are included as a reference. The solid lines represent the best fit to the experimental data. 
around the $\mathrm{Sn}$ atoms (e.g. defects, bond stretching, etc.). In contrast, for the as-grown, amorphous sample a good fit could only be obtained with an even smaller path degeneracy $(\mathrm{N}<3)$ to account for the reduced peak intensity. This implies that the $\mathrm{Sn}$ atoms are in a more defective, less ordered environment before crystallisation. The changes in local structural order upon annealing are also reflected in the $\mathrm{Sn}-\mathrm{Ge}$ distance. More specifically, the bond length is reduced from $2.65 \pm 0.05 \AA$ to $2.60 \pm 0.05 \AA$ after crystallization. This is comparable to the reduction in $\mathrm{Si}-\mathrm{Ge}$ bond length $(\sim 0.02 \AA)$ observed upon crystallization of amorphous $\mathrm{SiGe}$ films with $5 \% \mathrm{Si}$ concentration ${ }^{24}$ or the reduction in Ge-Ge bond length $(\sim 0.02 \AA)$ of crystalline $\mathrm{Ge}$ films compared to amorphous Ge ${ }^{25}$ Furthermore the measured $\mathrm{Sn}-\mathrm{Ge}$ distance of $2.65 \pm 0.05 \AA$ in the amorphous GeSn film is in good agreement with the reported value of $2.67 \AA$ for a-GeSn films on an Al foil. ${ }^{25}$ Due to the larger atom size of $\mathrm{Sn}$ with respect to $\mathrm{Ge}$, the bond length of the crystallized c-GeSn(4.5\%) sample is yet larger than that of a pure Ge crystal $(2.45 \AA)$.

Figure $3 b$ shows the magnitude of the FT for the amorphous a$\operatorname{GeSn}(11.3 \%)$ and the crystalline c-GeSn(11.3\%) samples. The structure of the as-grown $11.3 \%$ sample is comparable to that of the asgrown $4.5 \%$ sample, with a Sn-Ge distance of $2.64 \pm 0.05 \AA$ and a similar defective environment, hence a lower local order around the $\mathrm{Sn}$ atoms than in the ideal crystal structure. This finding is consistent with the results reported in Ref. 25: in their experimental study the authors likewise found a Sn-Ge distance $(2.67 \AA)$ for amorphous GeSn films which is invariant with Sn concentration (for up to 20\%). In contrast to the $4.5 \% \mathrm{Sn}$ sample, we observe a decrease in peak height after annealing, which can be linked to a further decrease in structural order around the $\mathrm{Sn}$ atoms and/or a decrease in the amount of substitutional $\mathrm{Sn}$. In addition, a second component is clearly visible at approximately $3 \AA$ after annealing the $11.3 \%$ sample. We assign this feature to Sn-Sn scattering based on a comparison to the metallic Sn reference. The XANES region (Figure 4) likewise confirms the formation of metallic Sn agglomerates (i.e. Sn-Sn bonds) after annealing. Unlike the as-grown sample, the XANES fine structure of the annealed sample resembles that of a metallic $\beta$-Sn reference. Annealing the a-GeSn $(11.3 \%)$ sample at $500^{\circ} \mathrm{C}$ therefore leads to a reduction in substitutional $\mathrm{Sn}$, hence to the formation of $\mathrm{Sn}$ clusters. To fit the EXAFS data of the annealed $11.3 \%$ sample, both a Sn-Ge and a Sn-Sn single scattering path were used with independent bond distance $(\Delta \mathrm{R})$ values for each path. The extracted bond lengths are $2.59 \pm 0.05 \AA$ for the $\mathrm{Sn}-\mathrm{Ge}$ and $3.01 \pm 0.05 \AA$ for the $\mathrm{Sn}-\mathrm{Sn}$ bonds.

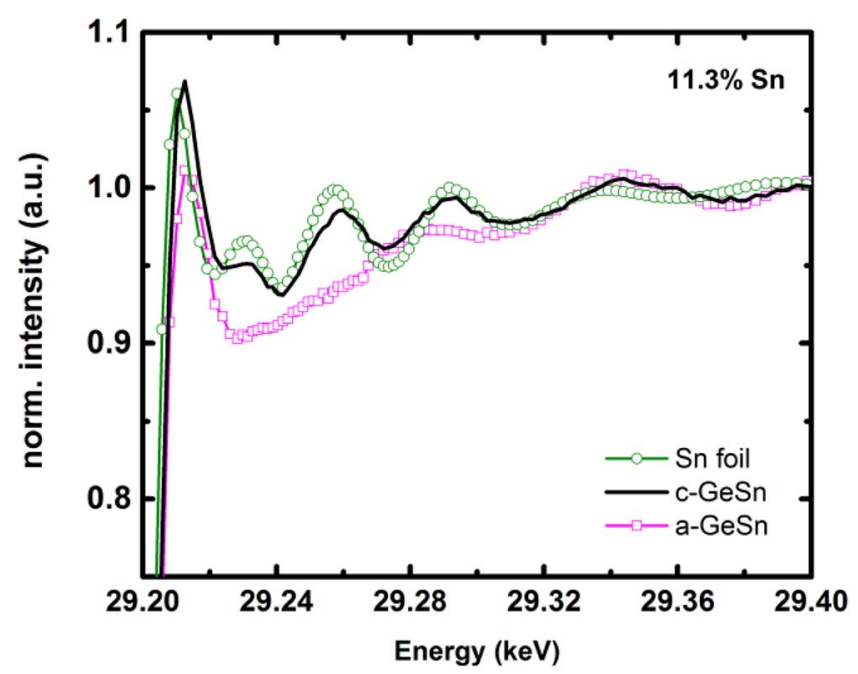

Figure 4. XANES data around the Sn K-edge (29.2 keV) of crystalline (black solid line) and amorphous (pink line with squares) GeSn with nominally $11.3 \%$ Sn. A metallic Sn reference foil (green line with circles) is included as a reference.
Similar to the nominally $4.5 \%$ Sn sample, the Sn-Ge bond length decreases after annealing the $11.3 \% \mathrm{Sn}$ sample. The Sn-Ge bond length of the annealed structure c-GeSn(11.3\%) compares very well with the mean Sn-Ge bond length $(2.58 \AA)$ obtained from $a b$-initio calculations by Beeler et al. ${ }^{26}$ for a diamond structure $\mathrm{Ge}_{0.9} \mathrm{Sn}_{0.1}$ alloy. The same theoretical study ${ }^{26}$ reports a characteristic $\mathrm{Sn}-\mathrm{Sn}$ bond length of $2.711 \AA$. This value is, however, significantly smaller than the value found experimentally in our study (3.01 $\AA$ ), but it compares well to the theoretically calculated $\mathrm{Sn}$-Sn bond length in $\alpha$-Sn $\left(2.796 \AA^{26}\right)$. In contrast, for $\beta$-Sn a Sn-Sn bond length of $3.016 \AA$ is reported, ${ }^{27}$ which is identical to our EXAFS results. Hence, our XAS measurements show conclusively that metallic $\beta$-Sn clusters are formed in the $11.3 \%$ Sn sample after crystallization at $500^{\circ} \mathrm{C}$.

Further improvement of the solid phase epitaxy method is therefore required for GeSn layers with high $\mathrm{Sn}$ content $(>6 \%){ }^{15}$ For increasing Sn content the probability of forming small Sn clusters during the deposition increases. Such Sn clusters can lead to preferential crystallization during the annealing step and therefore result in crystal defects and inferior structural quality. Suppressing the formation of Sn clusters during deposition can be achieved by decreasing the adatom diffusion length. The surface diffusion length $\lambda_{S}$ can be described by the following equation: $\lambda_{S}=a e^{\left(-E_{D i f f} / 2 k T\right)} \sqrt{\nu N_{S} / J_{G e}}$ with $\nu, a, T, E_{D i f f}, N_{S}$ and $J_{G e}$ the hopping attempt rate, the nearestneighbor distance, the substrate temperature, the surface diffusion activation energy, the density of available surface lattice sites and the incident Ge flux, respectively. Decreasing the deposition temperature and increasing the growth rate is very effective for reducing the diffusion length and is therefore expected to lead to improved structural quality for GeSn layers with high Sn content ( $>6 \%$ ). Clustering of Sn atoms during deposition is an even more important issue for conventional GeSn epitaxy. When comparing the adatom diffusion length of conventional epitaxy with the atom diffusion length of SPE, the potential of SPE for GeSn becomes very apparent, as will be discussed in the following paragraph. As a diffusion coefficient for $\mathrm{Sn}$ on $\mathrm{Si}$ or $\mathrm{Sn}$ on Ge we take the value of diffusion of Ge on Si $\left(2.53 \times 10^{-7}\right.$ $\left.\exp (-0.676 \pm 0.03 \mathrm{eV} / \mathrm{kT}) \mathrm{cm}^{2} / \mathrm{s}^{28}\right)$ because no specific information has been reported. Although there might be a significant difference in the real diffusion coefficient, we can use these values to obtain a qualitative interpretation and comparison between SPE and conventional epitaxy. For conventional GeSn epitaxy with chemical vapor deposition (CVD) the typical deposition temperature is around $300^{\circ} \mathrm{C}$ with a deposition rate of $100 \mathrm{~nm} / \mathrm{h},{ }^{14}$ leading to an adatom diffusion length of $\sim 800 \mathrm{~nm}$. For the SPE method much lower deposition temperatures and higher growth rates can be used. For a deposition temperature of RT and growth rate of $1 \mu \mathrm{m} / \mathrm{h}$, the adatom diffusion length is only $\sim 0.3 \mathrm{~nm}$. For the SPE process we also need to take into account diffusion of Sn atoms in the layer during the annealing step. For the diffusion coefficient $D$ of $\mathrm{Sn}$ in Ge we use the reported value of 70 $\exp (-3.05 \mathrm{eV} / \mathrm{kT}),{ }^{29}$ from which we can calculate the bulk diffusion length $\lambda_{B}: \lambda_{B}=\sqrt{D t}$. For a relatively high annealing temperature of $600^{\circ} \mathrm{C}$ and annealing time of $1 \mathrm{~min}$, the Sn bulk diffusion length $\lambda_{B}$ is limited to $\sim 1 \mathrm{~nm}$. The total diffusion length (surface diffusion and bulk diffusion) is therefore expected to be much lower for SPE compared to conventional epitaxy. Table II summarizes the comparison of diffusion lengths for SPE and conventional epitaxy. Diffusion at the surface can still be significant in the case of SPE during the annealing

\section{Table II. Comparison diffusion lengths for GeSn in solid phase} epitaxy and conventional epitaxy.

\begin{tabular}{lcc} 
& SPE & Epitaxy \\
\hline Technology & PE-CVD + RTA & CVD \\
Deposition temp. & RT & $300^{\circ} \mathrm{C}$ \\
Growth rate & $1 \mathrm{micron} / \mathrm{h}$ & $100 \mathrm{~nm} / \mathrm{h}$ \\
Surface diffusion length $\lambda_{S}(\mathrm{~nm})$ & $\sim 0.3$ & $\sim 800$ \\
Diffusion length $\lambda_{B}$ during $1^{\prime} 600^{\circ} \mathrm{C}$ & $\sim 1$ & n.a.
\end{tabular}



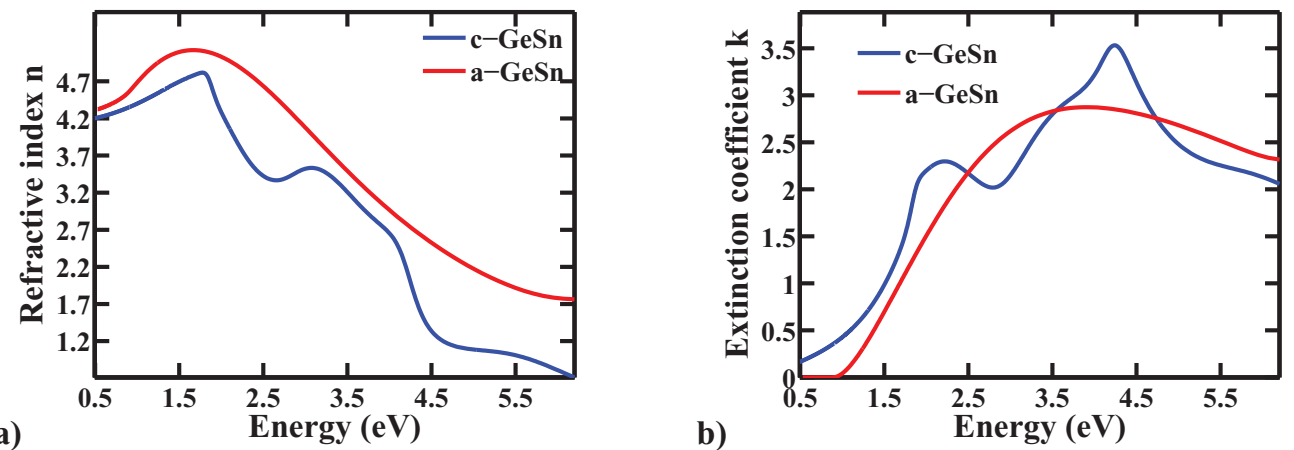

Figure 5. a) Refractive index $n$ and b) extinction coefficient $k$ of amorphous and crystalline GeSn with nominally $4.5 \%$ Sn, measured by spectroscopic ellipsometry.

step. However this can be suppressed by capping the surface before annealing with an immobile layer, e.g. a dielectric layer, Si or Ge. In general the adatom diffusion length $\lambda_{S}$ will decrease with a factor of $\sqrt{x}$ for an $x$-fold increase in deposition rate, and will decrease with a factor $e^{y}$ for a $y$-fold decrease in temperature. Reducing the temperature, e.g. using cryogenic temperatures, is therefore more effective than increasing the deposition rate. Additional to using low deposition temperatures and high deposition rates, plasma enhanced CVD can be used to decrease the diffusion length during deposition by supplying reactive hydrogen atoms. ${ }^{16}$

It is expected that the structural differences between the amorphous and crystallized GeSn layer have a significant impact on the physical properties. We therefore assess the optical properties of asdeposited (amorphous) and annealed (crystalline) GeSn samples of $37 \mathrm{~nm}$ thickness and $4.5 \%$ Sn content by spectroscopic ellipsometry. As mentioned before, a crystalline and tensile strained $\mathrm{GeSn}$ layer with $4.5 \% \mathrm{Sn}$ showed a direct transition in transmission measurements. ${ }^{15}$ The spectroscopic ellipsometer used in this work (SENresearch SE 850 DUV) includes a Fourier transform infrared (FTIR) spectrometer for measurements in the NIR spectral range, allowing measurements from $200 \mathrm{~nm}(6.2 \mathrm{eV})$ up to $2500 \mathrm{~nm}(0.5 \mathrm{eV})$. As the $11.3 \%$ sample contains Sn segregates, no optical measuremens have been undertaken on this sample set. We have fitted the refractive index $n$ and extinction coefficient $k$ extracted from the data, see Figure $5 \mathrm{a}$ and $5 \mathrm{~b}$, respectively. The optical properties show a significant change upon crystallization. Previously we determined the bandgap energy of a $300 \mathrm{~nm}$ crystalline GeSn film deposited with a $4.5 \% \mathrm{Sn} /(\mathrm{Sn}+\mathrm{Ge})$ flux ratio on $\mathrm{Si}$ by transmission measurements and found an optical bandgap of $0.52 \mathrm{eV}(2383 \mathrm{~nm}) \pm 0.05 \mathrm{eV}$ with direct transition. ${ }^{15}$ The presence of tensile strain in our GeSn layer lowers the difference between the direct and indirect transition in GeSn and is therfore beneficial for obtaining a direct bandgap material. Thanks to this tensile strain $(+0.34 \%$ for $4.5 \% \mathrm{Sn})$ our layers show a direct transition for a Sn composition $(4.5 \%)$ which is lower than for unstrained $(6.4 \%)$ or compressively strained GeSn. ${ }^{30}$

For the amorphous GeSn sample investigated in this work, we followed a similar approach: the absorption coefficient $\alpha$ was calculated from the extinction coefficient $k$ and the incident wavelength $\lambda_{0}$ in free space as: $\alpha\left(\lambda_{0}\right)=4 \pi k / \lambda_{0}$. Subsequently we fitted the absorption coefficient $\alpha$ to the equation $\alpha \boldsymbol{h} \boldsymbol{v}=\boldsymbol{C}_{\boldsymbol{A}}\left(\boldsymbol{h} \boldsymbol{v}-\boldsymbol{E}_{g}\right)^{n}$ with $h v$ the photon energy, $C_{A}$ a constant that differs for different transitions, $n$ is an index which depends on the transition, and $E_{g}$ the Tauc gap. This Tauc gap is used as a measure of the optical gap or bandgap in amorphous materials. For the amorphous GeSn sample we find an optical gap of $0.89 \mathrm{eV}$ $(1392 \mathrm{~nm}) \pm 0.05 \mathrm{eV}$. Furthermore, $n=2$, is deduced, which indicates that the transition requires momentum transfer through phonons ${ }^{31}$ and is therefore indirect. In other words, after crystallization the optical properties of the GeSn layer change from an indirect to a direct transition and the bandgap decreases significantly. This property is useful in the fabrication of optical switches or memory. ${ }^{32}$ The penetration depth of amorphous and crystalline GeSn with nominally $4.5 \% \mathrm{Sn}$ has been deduced from the extinction coefficient measured by spectroscopic

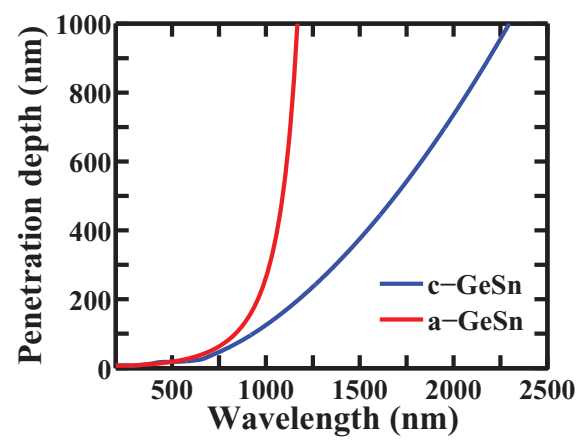

Figure 6. Penetration depth of amorphous and crystalline GeSn with nominally $4.5 \% \mathrm{Sn}$, calculated from the extinction coefficient measured by spectroscopic ellipsometry.

ellipsometry, see Figure 6. The penetration depth of light is defined as the distance at which the amplitude of the intensity of the light with wavelength $\lambda$ has been decreased to $1 / e(37.3 \%)$ of the incident intensity. The penetration depth can be calculated from the extinction coefficient $k$ by the following equation: $L=\frac{\lambda_{0}}{4 \pi k}$ with $\lambda_{0}$ being the wavelength of light in vacuum. The crystalline GeSn layer has a significantly lower penetration depth compared to the amorphous layer for wavelengths above $750 \mathrm{~nm}$, see Figure 6. This property is advantageous for high speed optical devices.

\section{Conclusions}

Improving our understanding of the fundamental aspects of SPE was the initial goal of this study. For his reason we have measured the structural properties of the as deposited amorphous GeSn film as well as the annealed film after solid phase epitaxy (crystallization), using Raman, RSM HR-XRD, XAS and spectroscopic ellipsometry. The measurement techniques used in this work are clearly complementary and help to understand the underlying physical mechanisms of the SPE process in GeSn layers. The Raman measurements yield particular information about the local order of $\mathrm{Ge}$ atoms and $\mathrm{Ge}-\mathrm{Ge}$ bonds; the XRD measurements yield the distance between crystalline planes, hence an average of both homonuclear and heteronuclear bond lengths; the RBS measuremens give information on the composition profile; whereas the XAS measurements are sensitive to the local environment and chemistry of the Sn atoms. The structural order of the sample increases significantly after annealing with respect to the as-deposited layer, which follows from solid phase crystallization of the amorphous GeSn. Thermal biaxial tensile strain is built into the layer during cool-down. A clear difference in the local structure of $\mathrm{Ge}$ and $\mathrm{Sn}$ is observed between the amorphous and crystalline layers. The Ge-Sn bond length decreases after annealing in both the $4.5 \%$ and the $11.3 \%$ nominal samples. For both the amorphous as well as the crystalline GeSn, the bond length is increased compared to 
bulk crystalline Ge. The Sn appears to be substitutional for the asdeposited and crystallized GeSn with nominally $4.5 \% \mathrm{Sn}$ and no $\mathrm{Sn}$ clustering was observed. For the as-deposited GeSn with nominally $11.3 \% \mathrm{Sn}$, no Sn segregation is observed. However after annealing at $500^{\circ} \mathrm{C}, \beta-\mathrm{Sn}$ clusters can be observed by EXAFS as well as a more inhomogeneous composition profile. From XRD it is clear that a significant part of the Sn does not incorporate substitutionally for the crystalline layer. When comparing the adatom diffusion length of conventional epitaxy with the atom diffusion length of SPE, the potential of SPE for GeSn becomes clear: the total diffusion length (surface diffusion and bulk diffusion) is expected to be much lower for SPE compared to conventional epitaxy. Additional to using low deposition temperatures and high deposition rates, plasma enhanced CVD can be used to decrease the diffusion length during deposition by supplying reactive hydrogen atoms. These measures are expected to suppress Sn clustering significantly and lead to an increase in the maximal achievable substitutional Sn concentration. Finally we determined the optical properties of amorphous and crystalline GeSn with nominally $4.5 \% \mathrm{Sn}$. The absorption of the GeSn layer changes from an indirect transition for the amorphous structure to a direct transition for the crystalline structure. The bandgap energy decreases significantly from $0.89 \mathrm{eV}(1392 \mathrm{~nm}) \pm 0.05 \mathrm{eV}$ for the amorphous layer to $0.52 \mathrm{eV}(2383 \mathrm{~nm}) \pm 0.05 \mathrm{eV}$ for the crystalline GeSn. The crystalline GeSn shows a significant lower penetration depth compared to the amorphous GeSn for wavelengths above $750 \mathrm{~nm}$.

\section{Acknowledgments}

Access to the DUBBLE beamline at ESRF has been granted by the Research Foundation Flanders (FWO) Big Science project, GOA/14/007 and SPIRIT are acknowledged for support. Y. Shimura acknowledges support from the Research Foundation Flanders (FWO) as Pegasus Marie Curie Fellow. C.F. has received funding through the KU Leuven BOF research program (PDMK/11/150).

\section{References}

1. J. D. Sau and M. L. Cohen, Phys. Rev. B 75, 045208 (2007).

2. S. Gupta, R. Chen, B. Magyari-Kope, H. Lin, B. Yang, A. Nainani, Y. Nishi, J. S. Harris, and K. C. Saraswat, Tech. Dig. - Int. Electron Devices Meet., 2011 , p. 16.6.1.

3. J. Kouvetakis, J. Menendez, and A. V. G. Chizmeshya, Annu. Rev. Mater. Res 36, 497 (2006).

4. R. Kotlyar, U. E. Avci, S. Cea, R. Rios, T. D. Linton, K. J. Kuhn, and I. A. Young, Appl. Phys. Lett. 102, 113106 (2013).
5. S. Gupta, B. Magyari-Kope, Y. Nishi, and K. C. Saraswat, J. Appl. Phys. 113, 073707 (2013).

6. J. Werner, M. Oehme, M. Schmid, M. Kaschel, A. Schirmer, E. Kasper, and J. Schulze: Appl. Phys. Lett. 98, 061108 (2011).

7. G. Han, S. Su, C. Zhan, Q. Zhou, Y. Yang, L. Wang, P. Guo, W. Wei, C. P. Wong, Z. X. Shen, B. Cheng, and Y.-C. Yeo, Tech. Dig. - Int. Electron Devices Meet., 2011, p. $16.7 .1-16.7 .3$.

8. G. He and H. A. Atwater, Appl. Phys. Lett. 68, 664 (1996).

9. Y.-Y. Fang, J. Tolle, R. Roucka, A. V. G. Chizmeshya, J. Kouvetakis, V. R. D'Costa, and J. Menéndez, Appl. Phys. Lett. 90, 061915 (2007).

10. S. Takeuchi, Y. Shimura, O. Nakatsuka, S. Zaima, M. Ogawa, and A. Sakai, Appl. Phys. Lett. 92, 231916 (2008).

11. C. D. Thurmond, F. A. Trumbore, and M. Kowalchik, J. Chem. Phys. 25, 799 (1956).

12. H. Pérez Ladrón de Guevara, A. G. Rodríguez, H. Navarro-Contreras, and M. A. Vidal, Appl. Phys. Lett. 84, 4532 (2004).

13. E. Kasper, J. Werner, M. Oehme, S. Escoubas, N. Burle, and J. Schulze, Thin Solid Films 520, 3195 (2012).

14. R. Loo, B. Vincent, F. Gencarelli, C. Merckling, A. Kumar, G. Eneman, L. Witters, W. Vandervorst, M. Caymax, M. Heyns, and A. Thean, ECS J. Solid State Sci. Technol. 2, 346 (2013)

15. R. R. Lieten, J. W. Seo, S. Decoster, A. Vantomme, S. Peters, K. C. Bustillo, E. E. Haller, M. Menghini, and J.-P. Locquet, Appl. Phys. Lett. 102, 052106 (2013).

16. R. R. Lieten, S. Degroote, F. Clemente, M. Leys, and G. Borghs, Appl. Phys. Lett. 96, 052109 (2010).

17. R. R. Lieten, T. Maeda, W. Jevasuwan, H. Hattori, N. Uchida, S. Miura, M. Tanaka, and J.-P. Locquet, Appl. Phys. Express 6, 101301 (2013).

18. H. Lin, R. Chen, Y. Huo, T. I. Kamins, and J. S. Harris: Appl. Phys. Lett. 98, 261917 (2011).

19. V. R. D'Costa, J. Tolle, R. Roucka, C. D. Poweleit, J. Kouvetakis, and J. Menendez, Solid State Communications 144, 240 (2007).

20. B. Ravel and M. Newville, J. Synchrotron Radiat. 12, 537 (2005).

21. M. Newville, J. Synchrotron Radiat. 8, 322 (2001).

22. J. J. Rehr and R. C. Albers, Phys. Rev. B 41, 8139 (1990).

23. S. I. Zabinsky, J. J. Rehr, A. Ankudinov, R. C. Albers, and M. J. Eller, Phys. Rev. B 52, 2995 (1995).

24. M. C. Ridgway, K. M. Yu, C. J. Glover, G. J. Foran, C. Clerc, J. L. Hansen, and A. Nylandsted Larsen, Phys. Rev. B 60, 10831 (1999).

25. S. Pascarelli, F. Boscherini, S. Mobilio, A. R. Zanatta, F. C. Marques, and I. Chambouleyron, Phys. Rev. B 46, 6718 (1992).

26. R. Beeler, R. Roucka, A. V. G. Chizmeshya, J. Kouvetakis, and J. Menéndez, Phys. Rev. $B$ 84, 035204 (2011).

27. W. Martienssen and H. Warlimont, Springer Handbook of Condensed Matter and Materials Data (Springer, Berlin, 2005) edited by Werner Martienssen and Hans Warlimont.

28. H. J. Kim, Z. M. Zhao, J. Liu, V. Ozolins, J. Y. Chang, and Y. H. Xie, J. Appl. Phys. 95, 6065 (2004)

29. C. Claeys and E. Simoen, Germanium-Based Technologies: From Materials to Devices, 1st ed. (Elsevier, New York, 2007).

30. H. Lin, R. Chen, W. Lu, Y. Huo, T. I. Kamins, and J. S. Harris, Appl. Phys. Lett. 100, 102109 (2012).

31. R. J. Elliott, Phys. Rev. 108, 1384 (1957).

32. A. V. Kolobov, P. Fons, A. I. Frenkel, A. L. Ankudinov, J. Tominaga, and T. Uruga, Nature Materials 3, 703 (2004). 